\title{
Yes-activated protein promotes primary resistance of BRAF V600E mutant metastatic colorectal cancer cells to mitogen-activated protein kinase pathway inhibitors
}

\author{
Meng Su ${ }^{1}$, Lei Zhan ${ }^{1}$, Yong Zhang ${ }^{2}$, Jingdong Zhang ${ }^{1}$ \\ ${ }^{1}$ Medical Oncology Department of Gastrointestinal Cancer, Cancer Hospital of China Medical University, Liaoning Cancer Hospital \& Institute, \\ Shenyang, China; ${ }^{2}$ Department of Pathology, Cancer Hospital of China Medical University, Liaoning Cancer Hospital \& Institute, Shenyang, China \\ Contributions: (I) Conception and design: M Su, J Zhang; (II) Administrative support: None; (III) Provision of study materials or patients: None; (IV) \\ Collection and assembly of data: M Su, L Zhan; (V) Data analysis and interpretation: All authors; (VI) Manuscript writing: All authors; (VII) Final \\ approval of manuscript: All authors. \\ Correspondence to: Jingdong Zhang. No. 44 Xiaoheyan Road, Dadong District, Shenyang 110042, China. Email: jdzhang@cancerhosp-ln-cmu.com.
}

Background: Most colorectal cancer (CRC) patients with the BRAF V600E mutation display resistance to chemotherapy and targeted medicinal treatments. Thus, exploring new drugs and drug resistance mechanisms for the BRAF V600E mutation has become an urgent clinical priority.

Methods: MTS experiment, cell cloning experiment, cell scratching experiment, Transwell experiment, chromatin immunoprecipitation (ChIP), quantitative polymerase chain reaction (qPCR) and flow cytometry are used. Detect the transcription and protein expression of YAP in colorectal cancer cell lines, establish a transient cell line with YAP gene overexpression and knockdown, and detect the effect of YAP gene expression on the biological functions of colorectal cancer cells RKO and HT-29. And further study the mechanism of YAP regulating the response of $R A F$ and $M E K$ targeted therapy.

Results: In this study, for the first time, we verified that the expression of transcription factor yes-associated protein (YAP) was upregulated in BRAF V600E mutant CRC cells. After knocking down YAP, we observed a reduction in the growth rate, proliferation, and invasion ability of colon cancer cells. We further verified that YAP knockdown increased sensitivity of $B R A F$ V600E mutant CRC cells to mitogen-activated protein kinase (MAPK) pathway inhibitors. In addition, we clarified the mechanism underlying YAP regulation of $R A F$ and MAPK/extracellular signal-regulated kinase $(M E K)$-targeted therapy response: YAP cooperates with $R A F \rightarrow M E K$ pathway inhibitors to regulate the cell cycle, increase cell G1/S phase arrest, and increase apoptosis.

Conclusions: These results suggest that YAP expression may be related to the primary resistance of MAPK inhibitors in metastatic CRC with the BRAF V600E mutation. Therefore, the combination of YAP and MAPK pathway inhibitors in BRAF V600E mutant metastatic CRC may present a promising treatment method.

Keywords: Colorectal cancer (CRC); BRAF V600E mutation; yes-activated protein; mitogen-activated protein kinase pathway (MAPK pathway)

Submitted Apr 06, 2021. Accepted for publication Jun 11, 2021.

doi: 10.21037/jgo-21-258

View this article at: https://dx.doi.org/10.21037/jgo-21-258

(c) Journal of Gastrointestinal Oncology. All rights reserved. 


\section{Introduction}

Colorectal cancer (CRC) has the sixth highest incidence and third highest mortality among cancers in China $(1,2)$, and approximately $15 \%$ of patients with metastatic CRC have $B R A F$ gene mutations and poor prognosis $(3,4)$. $B R A F$ oncogenes promote tumorigenesis by activating the mitogen activated protein kinase (MAPK) pathway (5), and the V600E mutation is the most common $B R A F$ mutation. The risk of death is twice as high in patients with the V600E mutation than in patients with wild-type $B R A F$ because most individuals in the former group are resistant to chemotherapy and targeted medicinal treatments. Although some small-sample studies have shown that three-drug chemotherapy (FOLFOXIRI) combined with bevacizumab may improve the prognosis of these patients to some extent, the survival benefit appears limited $(6,7)$. Thus, the exploration of novel drugs and treatment regimens for $B R A F$ V600E mutation constitutes an urgent clinical priority.

Yes-associated protein (encoded by YAP1) is a transcriptional co-activator which has recently emerged as a critical oncogene in multiple cancers, with increasing attention being paid to its biology and regulation (8). Studies have shown that activated YAP can promote resistance to $R A F, M A P K /$ extracellular signal-regulated kinase $(M E K)$, and epidermal growth factor receptor $(E G F R)$ inhibitors in a variety of cancer cell lines and cancer patients with $B R A F, K R A S$, and EGFR mutations $(9,10)$. The YAP transduction pathway is associated with the acquisition of drug resistance in melanoma, non-small cell lung cancer, breast cancer, and CRC (11). Additionally, YAP expression is negatively associated with cetuximab sensitivity in CRC cell lines independent of KRAS mutation status, and YAP knockdown enhances cetuximab cytotoxicity (12).

Here, we first examined the effects of YAP on the malignant biological behavior of $B R A F \mathrm{~V} 600 \mathrm{E}$ mutant colon cancer cells, as well on the sensitivity of mutant RKO and HT29 cells to MAPK (RAS-RAF-MEK-ERK) pathway inhibitors. We found that YAP can promote the primary resistance of $B R A F$ V600E mutant CRC cells to MAPK pathway inhibitors. Furthermore, we clarified the mechanism underlying YAP regulation of the $R A F$ - and $M E K$-targeted therapy response, providing new ideas and strategies for the clinical treatment of metastatic CRC and research on drug resistance. We present the following article in accordance with the MDAR reporting checklist (available at https://dx.doi.org/10.21037/jgo-21-258).

\section{Methods}

\section{Cell culture and agents}

The BRAF V600E mutant and human HCT116, SW480, SW48, Lovo, Caco2, HT29, and RKO were obtained from the Type Culture Collection of the Chinese Academy of Sciences (Shanghai, China). The cells were cultured RPMI1640 medium and Dulbecco's modified Eagle's medium (DMEM, Gibco, Thermo Fisher Scientific, Waltham, MA, USA) supplemented with $10 \%$ fetal bovine serum (Fc Gibco, Thermo Fisher Scientific) and 1\% penicillin/ streptomycin (Gibco, Thermo Fisher Scientific), and placed in a humidified incubator at $37^{\circ} \mathrm{C}$ and $5 \% \mathrm{CO} 2$.

\section{Small interfering RNA (siRNA) transfection}

The YAP siRNA and control siRNA were purchased from Shanghai GenePharma (Shanghai, China). The two independent siRNAs were used to target YAP: 5 ' -TTTCACTGGAGCACTCTGACT-3' (YAP 1) and 5'-TTTCACTGGAGCACTCTGACT-3' (YAP-2), and the control siRNA sequence was 5'-TCAGAGTGCTCCAGTGAAA-3'. Cells were transfected with control siRNA and YAP siRNA for $24 \mathrm{~h}$ by Lipofectamine 2000 (Invitrogen; Thermo Fisher Scientific, Waltham, MA, USA) for subsequent experiments.

\section{Western blot analysis}

The required proteins were obtained from cells by RIPA lysate and $10 \%$ sodium dodecyl sulfate-polyacrylamide gel (SDS-PAGE) electrophoresis. Western blots were probed with specific antibodies against CDK6, ERK, P-ERK (Cell Signaling Technology, CST), SGK1 (Hua An Organism), Bcl-2 (Abcam), Cyclin D1, YAP (Santa), $\beta$-actin (CST), LaminB1 (CST), PARP (CST), $\alpha$-Tubulin (CST), pRb (S807/811) (CST), E2F (CST), and Mcl-1 (CST). Blotting results were visualized using a chemiluminescent detection system (Pierce ECL Substrate Western Blot Detection System, Thermo Scientific, IL, USA) and exposure to autoradiography film (Kodak XAR).

\section{In vitro migration and invasion assays}

In vitro migration and invasion were detected by Transwell assay. The cells were cultured according to the number of $4 \times 10^{4}$ cells in each compartment for $72 \mathrm{~h}$, and the addition of matrix glue in the cells was to promote invasion. Cells in 
the chamber were swabbed, fixed with $100 \%$ methanol, and stained with $1 \%$ crystal violet for 20 minutes. The migrated and invaded cells were then quantified using a BX51 microscope (original magnification, $\times 100$ ).

\section{Flow cytometry}

Cell apoptosis was measured with an Annexin V-APC/PI Double staining apoptosis Detection Kit (Kaiji Biology, Jiangsu Province.) according to the manufacturer's instructions. In general, the treated cells were centrifuged, the supernatant was removed and retained, and the cells were resuspended in $195 \mathrm{~L} 1 \times$ Binding Buffer. Annexin $\mathrm{V}$-APC/PI $5 \mathrm{~L}$ and propidium iodide $10 \mathrm{~L}$ were added to the cell suspension, incubated in dark for $20 \mathrm{~min}$, and tested immediately. FACScan flow cytometry (BD Biosciences, San Jose, CA, USA) bioinformatics predicted apoptosis rates.

\section{Bioinformatics prediction}

The binding sites of the $S G K 1$ promoter and transcription factor YAP-TEAD Inhibitor 1 were predicted using the JASPAR (http://jaspar.genereg.net/) and miRDB (https://www.ncbi.nlm. nih.gov/) databases. We searched for downstream target genes activated by transcription of the YAP-TEAD complex.

\section{Statistical analysis}

Data were analyzed using SPSS Statistics version 25.0 software (IBM, Corporation, Armonk, NY). All results, except for the results from the western blot assay, were expressed as the mean value \pm standard deviation (SD) of triplicate independent experiments, and $\mathrm{P}$ values $<0.05$ were considered significant.

\section{Results}

YAP expression was upregulated in CRC cells with the BRAF V600E mutation

We used western blotting to quantify the transcript and protein expression of EGFR in eight CRC cell lines and found YAP expression levels were the highest in $B R A F$ V600E-mutated CRC cells (HT29 and RKO) (Figure 1).

\section{YAP knockdown inbibits the biological functions of RKO and HT29 in CRC cells with the BRAF V6OOE mutation}

To evaluate the effect of YAP on the malignant biological behavior of V600E mutant CRC cell lines, we used specific short hairpin RNAs (shRNAs) to inhibit YAP expression in those cells.

We transfected RKO cells with YAP plasmids (shYAPcontrol, shYAP1, shYAP2, and shYAP12), and HT29 cells with YAP lentivirus (shYAP-control and shYAP). Fluorescence electron microscopy showed sufficient infection, and western blotting verified knockdown results. MTS cell viability testing showed that the cell growth rate increased with greater YAP content. Similarly, cell cloning experiments identified stronger cell population dependence and proliferation ability with higher YAP content, and the cell scratch experiment confirmed these patterns. Transwell tests revealed a significant reduction in the invasion ability of YAP knockdown cells (Figure 1).

\section{YAP inbibits the sensitivity of BRAF V600E mutant CRC cells to $M A P K(R A S \rightarrow R A F \rightarrow M E K \rightarrow E R K)$ pathway inbibitors}

We used plasmid or viral shRNAs to knock out YAP in RKO and HT29CRC cells. YAP knockdown increased the sensitivity of RKO and HT29CRC cells to vemurafenib, trametinib, C225 + vemurafenib, and C225 + vemurafenib + trametinib, but had little effect on PBS-treated cells.

YAP knockdown had no significant effect on sensitivity to the cytotoxic chemotherapy drugs L-OHP and SN38, indicating that the silencing effect of YAP specifically targets the $R A S \rightarrow R A F \rightarrow M E K$ pathway (Figure $2 A, B, C$ ).

\section{YAP regulates the response mechanism of $R A F$ - and MEK- targeted therapy}

\section{Transcriptomics analysis}

We used transcriptomics to test differential gene expression between wild-type YAP-expressing HT29 cells and YAPknockdown HT29 cells. The results showed differential expression of multiple genes related to the cell cycle and FoxO signaling pathway (Figure 2D).

\section{Mechanism 1: BRAF, MEK inhibitors, and YAP have a} synergistic effect in regulating the cell cycle, increasing G1/S phase arrest

To further determine the mechanism underlying YAP regulation of $R A F$ and $M E K$ responses to targeted therapy, we conducted cell cycle-related experiments based on transcriptomics results. Flow cytometry revealed that YAP knockdown synergistically increased G1/S phase arrest in 
A

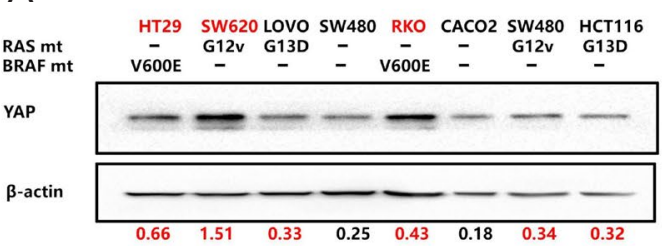

C

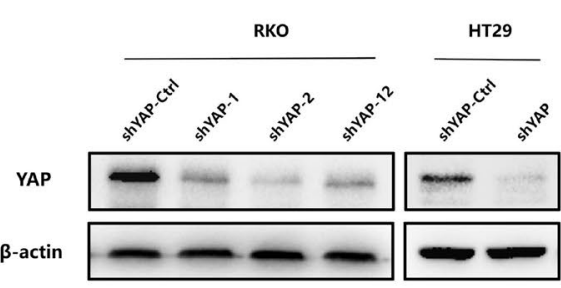

B
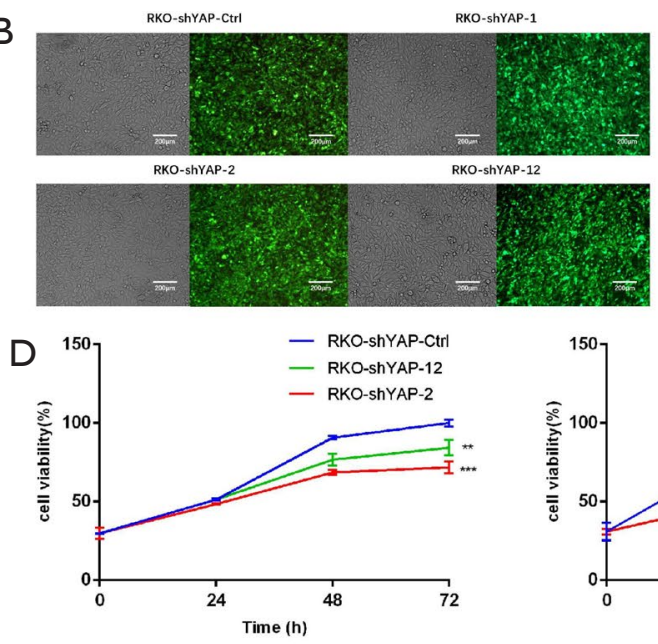

oh
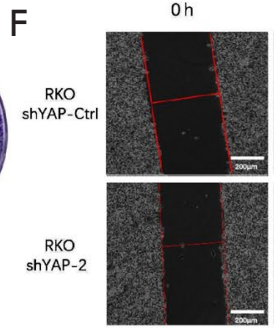

$\underset{\text { Sh`AP-12 }}{R=2}$

$24 \mathrm{~h}$
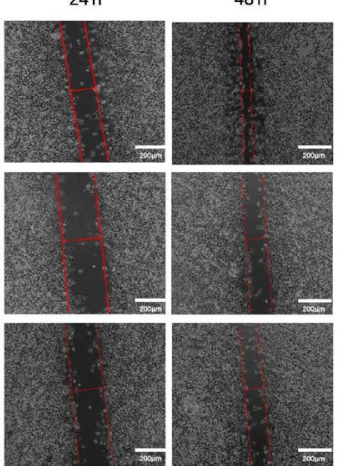
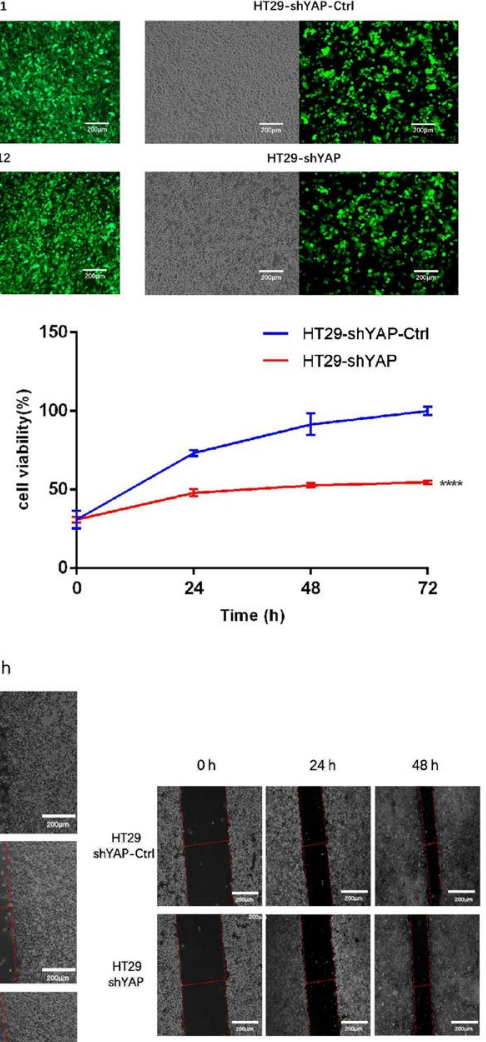

HT29

G

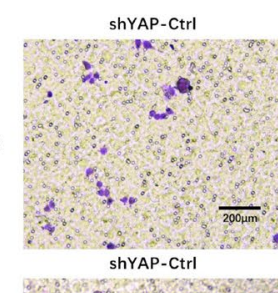

ShYAP-2

ShYAP-12
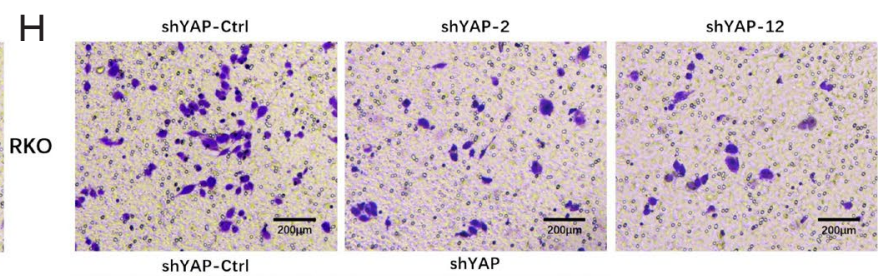

HT29

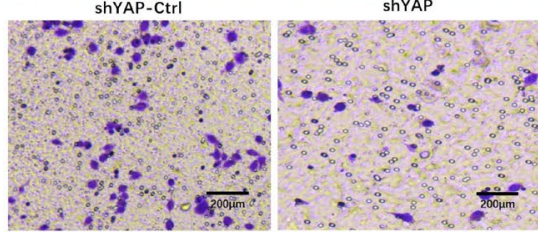

Figure 1 YAP depletion attenuates CRC cell proliferation, migration, and invasion and increases cell apoptosis in vitro. (A) In multiple CRC cell lines, YAP expression levels were detected by immunoblot using YAP-specific antibodies, and the results showed that high YAP levels were observed in BRAF V600E mutated CRC cells. (B,C) RKO was transfected with YAP plasmid for 24 hours, HT29 was transfected with YAP-shRNA virus for 24 hours, and fluorescence microscopy was used to verify the transfection results. YAP levels were then tested by Western blot analysis. (D) RKO was transfected with YAP plasmid and HT29 was transfected with yap-shrna virus for 24,48, and 72 hours. Cell viability was determined by MTS method. (E) Colony formation assays, in which 500 HT 29, or RKO cells were treated with shYAP for 7 days. Representative images. (F) The wound healing assay. Wound fields were photographed at 0,24 , and $48 \mathrm{~h}$ after wounding at three different sites. Representative images. (G,H) Transwell invasion assay was performed. Representative images of the invasive cells on the membrane. CRC, colorectal cancer; YAP, yes-associated protein. ${ }^{* *} \mathrm{P}<0.05$, ${ }^{* * *} \mathrm{P}<0.01$. ${ }^{* * * *} \mathrm{P}<0.001$. 

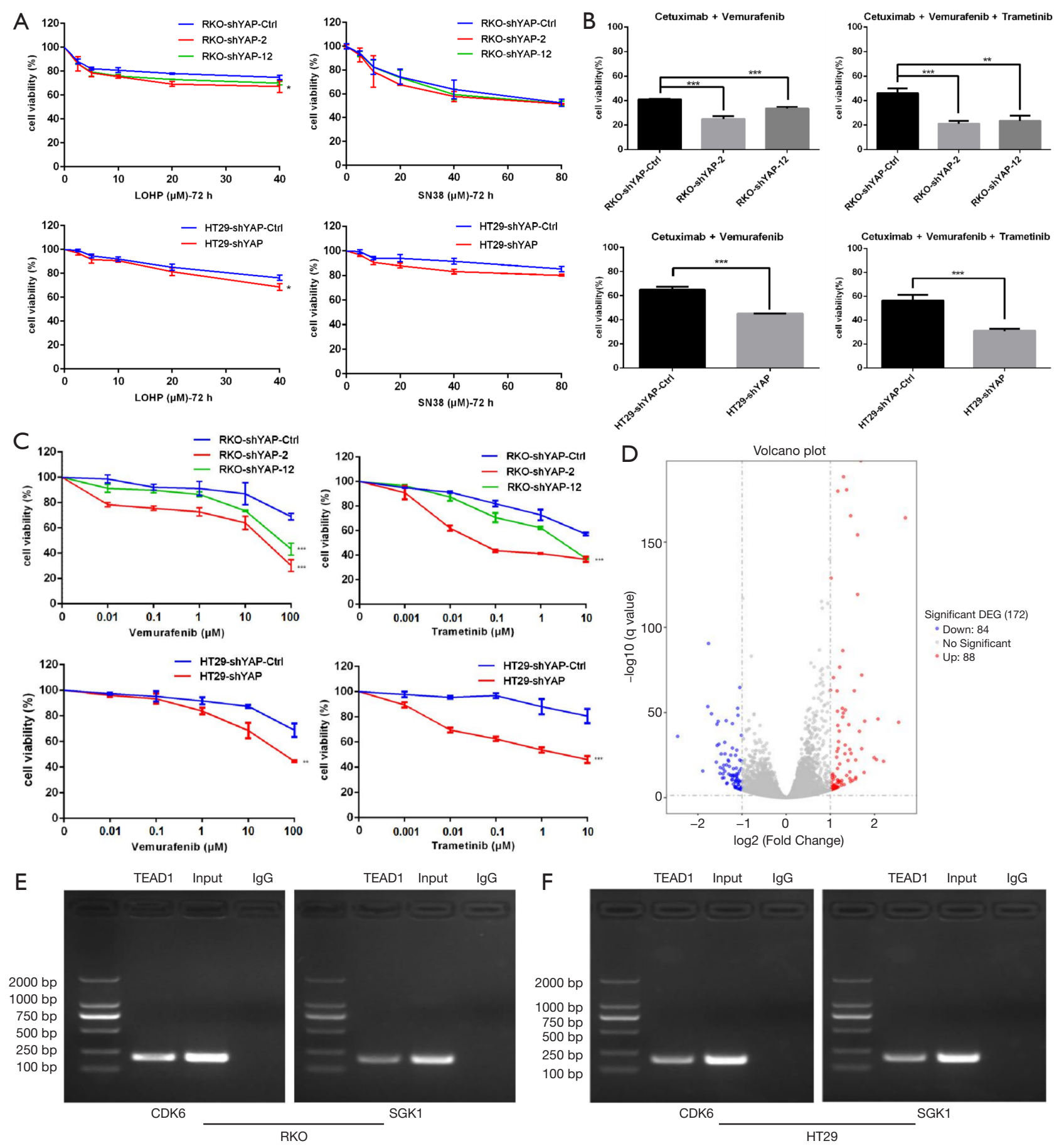

Figure 2 YAP inhibits the sensitivity of $B R A F$ V600E mutant CRC cells to MAPK $(R A S \rightarrow R A F \rightarrow M E K \rightarrow E R K)$ pathway inhibitors. (A,B) MTS method was used to detect the proliferation activity of cells. Knockdown of YAP increased the sensitivity of RKO and HT29 CRC cells to vemurafenib, trametinib, $\mathrm{C}+\mathrm{V}$, and $\mathrm{C}+\mathrm{V}+\mathrm{T}$, but had little effect on the cells treated with PBS. (C) Knockdown of YAP had no significant effect on the sensitivity of cytotoxic chemotherapeutic drugs L-OHP and SN38. (D) Transcriptomics tests the differential gene expression of HT29 cells that normally express wild-type YAP and YAP knockdown HT29 cells. The results show that the differential expression of multiple genes related to the cell cycle and FoxO signaling pathway. (E,F) CDK6 (cycle correlation) and SGK1 (FoxO pathway) genes are differentially expressed in these two pathways. Therefore, CHIP experiment was further performed to confirm the transcriptional regulation of YAP. ${ }^{*}, \mathrm{P}<0.1$. ${ }^{* *} \mathrm{P}<0.05,{ }^{* * *} \mathrm{P}<0.01$. CRC, colorectal cancer; YAP, yes-associated protein. 
the cell cycle of RKO and HT29 CRC lines treated with $R A F \rightarrow M E K$ pathway inhibitors.

\section{Mechanism 2: YAP knockdown downregulates the promoter CDK6 expression level, thus inhibits cell proliferation}

To regulate downstream target genes, the nonphosphorylated active form of YAP forms a complex with TEAD in the nucleus.

Previous studies found two conserved TEAD binding sites (GGAATG) in the human CDK6 promoter. We used chromatin immunoprecipitation (ChIP) and quantitative polymerase chain reaction (qPCR) to detect YAP-TEAD binding to these two sites and further confirmed that $C D K 6$ is the downstream target gene of the YAP-TEAD complex. Quantitative PCR and western blotting verified that CDK6 mRNA and protein were downregulated after YAP knockdown (13-18) (Figures 2E,F,3).

\section{Mechanism 3: SGK1 may be the downstream target gene of YAP transcriptional activation}

YAP positively regulates the downstream target gene $S G K 1$ to activate the $E R K 1 / 2$ pathway, reduce apoptosis induced by $R A F \rightarrow M E K$ pathway inhibitors, and increase RKO and HT29CRC resistance to those inhibitors.

We performed transcriptomics analysis on HT29 cells expressing wild-type YAP and shYAP, and consistent with previous microarray analyses, shYAP action significantly downregulated $S G K 1$ expression. Therefore, $S G K 1$ may be a downstream target gene of YAP transcription activation. We also used ChIP and qPCR to confirm that SKG1 is the downstream target gene of the YAP-TEAD complex. Experimental results show that $S G K 1$ is a potential positive feedback regulator of YAP and TAZ, and that the YAPTEAD/TAZ complex directly activates $S G K 1$ transcription by binding to the gene's distal enhancer.

Previous research demonstrated that SGK1 is an ERK1/2 activator and regulates the ERK1/2 pathway. Here, we treated RKO and HT29 cells with the SGK1 inhibitor EMD638683, then performed western blots and MTS to determine whether SGK1 regulates ERK1/2 activity. The experimental results showed that SGK1 inhibition significantly decreased pERK1/2 (activated form) expression, and there was no significant difference in the total amount between the two groups. Moreover, SGK1i and $\mathrm{RAF} \rightarrow$ MEK pathway inhibitors synergistically repress the ERK1/2 pathway, along with proliferation of RKO and HT29 cells (19-26).
$R A F \rightarrow M E K$ and $E R K$ pathways participate in apoptosis via regulating the gene expression of $B c l-2$ family proteins (e.g., Bax and Bim) and inducing proteasome degradation of antiapoptotic proteins. Flow cytometry detected apoptosis, and western blots revealed that YAP knockdown reduced $S G K 1$ expression. This in turn downregulated $p E R K$ expression, coordinating the expression of apoptosis related factors and increasing $R A F \rightarrow M E K$ induced apoptosis $(27,28)$ (Figure 4).

\section{Discussion}

The $R A S / R A F / M E K$ pathway is the first discovered and most thoroughly researched signaling pathway. In this, Ras protein activation triggers silk/threonine protein kinase to activate $R a f$ protein, which phosphorylates MEK1/2 regulatory serine to activate MEKs. Subsequently, $R A F$ selectively activates ERK, leading to antiangiogenic effects on cell proliferation and apoptosis. Raf kinase belongs to a protein family that includes Araf, BRAF, and Craf, along with their mutations (2), and Ras and $B R A F$ are mutually exclusive (29). The $B R A F$ gene is located in $7 \mathrm{q} 34$ and activates the MAPK signaling pathways downstream of the strongest agent. This is the codon 600 mutation (V600E), with a mutation rate above $90 \%$. Originally discovered as a marker of hairy cell leukemia, V600E is also found in other tumor tissues, including malignant melanoma, thyroid papillary carcinoma, CRC, pleomorphic xanthoastrocytoma, star cell tumors, cell tissue hyperplasia, borderline ovarian tumors, and glioma. The V600E mutation accounts for approximately $90 \%$ of all BRAF mutations. According to Chinese data approximately $5-10 \%$ of patients with metastatic CRC have BRAF mutations, and the incidence is approximately $5 \%$ in advanced CRC. Patients with the V600E mutation have a poor prognosis and with conventional treatment, have half the survival time of patients with wild-type $B R A F$.

A phase II clinical study compared the efficacy of chemotherapy + bevacizumab and chemotherapy + bevacizumab + cetuximab as first-line treatments for metastatic CRC. Subgroup analyses found that drug efficacy was unsatisfactory in both groups, with far lower median progression-free survival (PFS) and overall survival than that of wild-type patients (29). Another retrospective multivariate analysis studied disease progression in patients with metastatic CRC after receiving 5-fluorouracil-based chemotherapy. The results revealed that the BRAF V600E mutation was an independent predictor of poor prognosis 


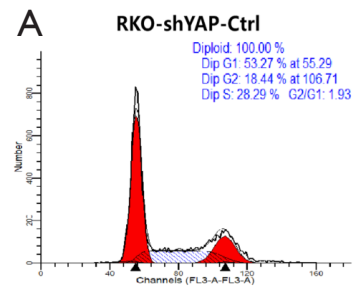

RKO-shYAP-2

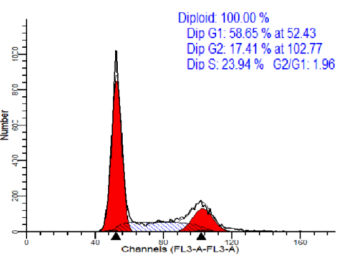

HT29-shYAP-Ctrl

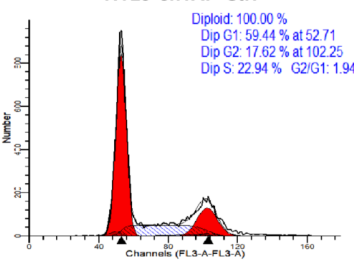

HT29-ShYAP
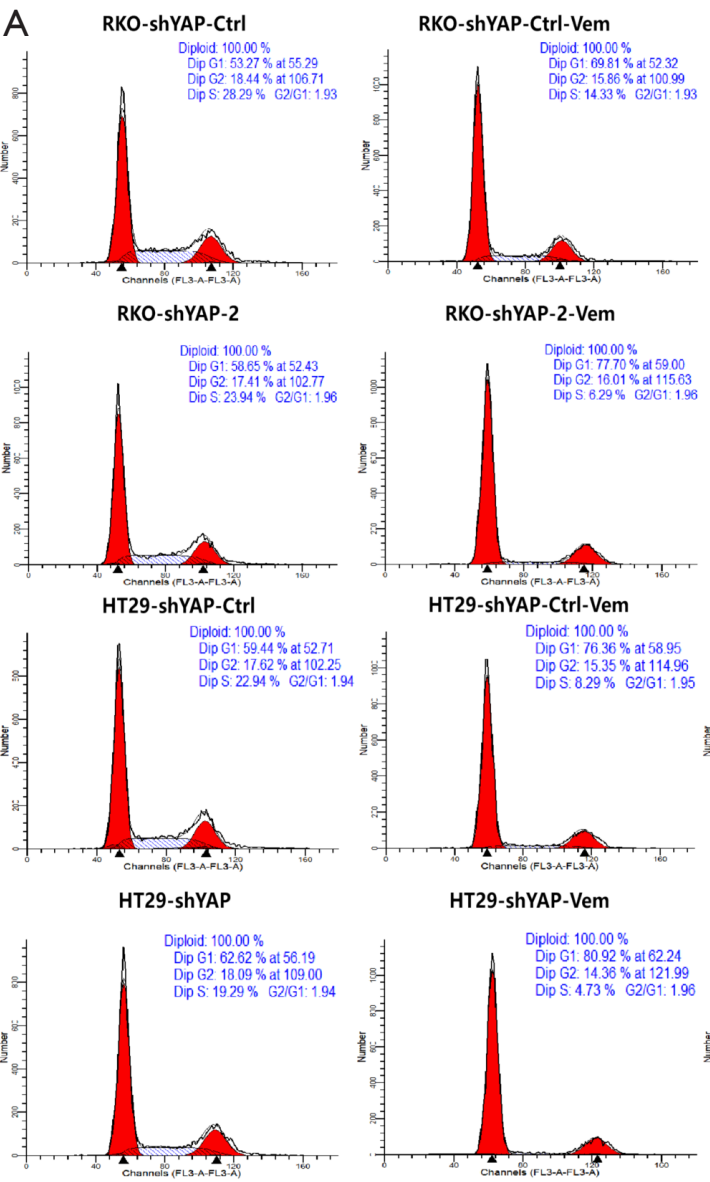

RKO-shYAP-2-Vem

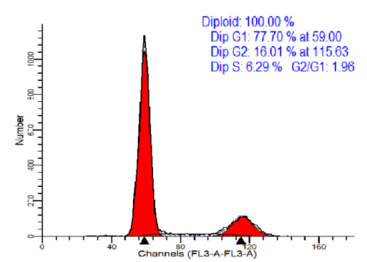

HT29-shYAP-Ctrl-Vem

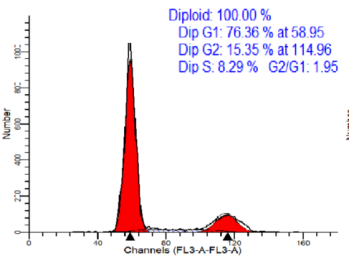

HT29-shYAP-Vem

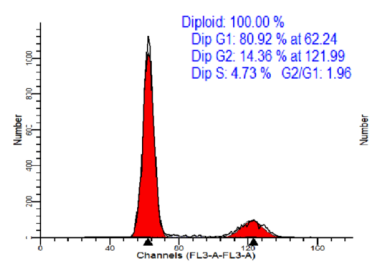

D

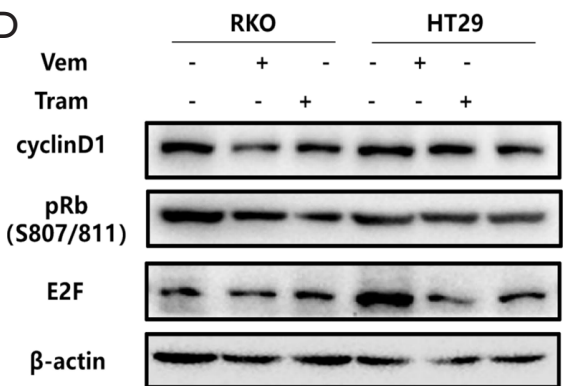

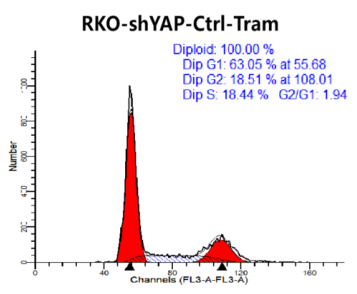

RKO-shYAP-2-Tram

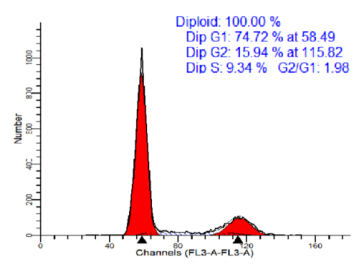

HT29-shYAP-Ctrl-Tram

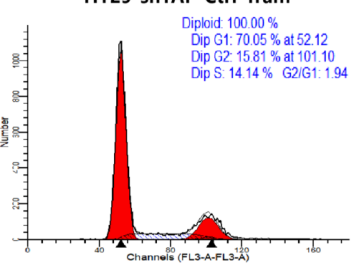

HT29-shYAP-Tram

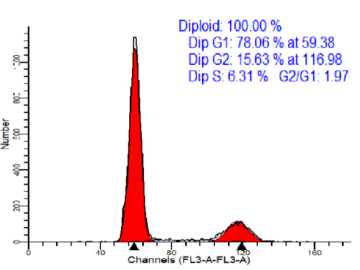

B
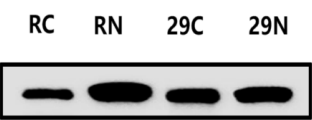

$\beta$-actin

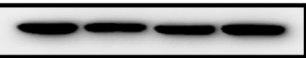

LaminB1 (Nuclear marker)

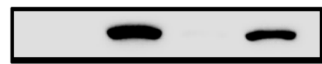

PARP (Nuclear marker)

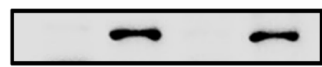

$\alpha$-Tubulin (Cytolsol marker)

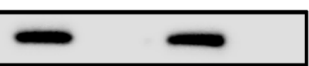

C

ShYAP

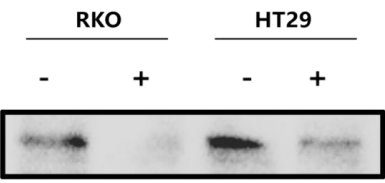

CDK6

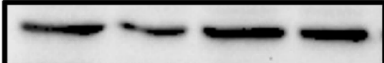

pRb

(S807/811)

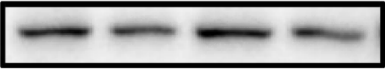

E2F

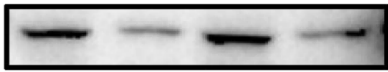

$\beta$-actin

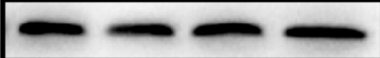

E
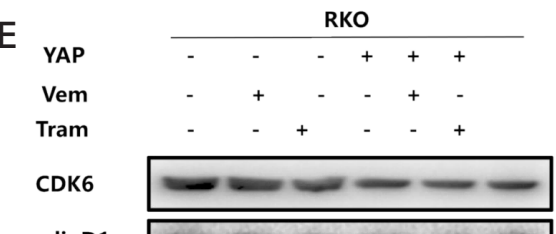

cyclinD1

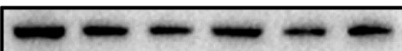

pRb
(S807/811)

E2F

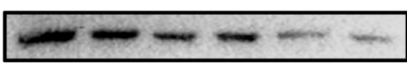

$-\infty--$

$-\infty-\infty-\infty$
HT29
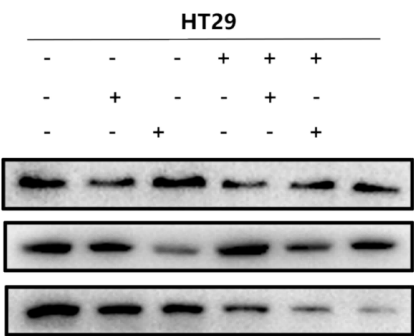

$-\infty-\infty-\infty$

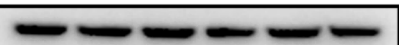

Figure $3 B R A F, M E K$ inhibitors, and YAP have a synergistic effect in regulating the cell cycle, increasing G1/S phase arrest. (A) Through flow cytometry to detect the cell cycle, we found that YAP knockdown can synergistically increase the G1/S phase block in the cell cycle of RKO and HT29CRC cells treated with $R A F \rightarrow M E K$ pathway inhibitors. (B) The nuclear expression of YAP in RKO and HT29CRC cells was observed in Western Blot. (C,D) WB confirmed that CDK6 was downregulated in both mRNA and protein levels after YAP knockdown. (E) BRAF and MEK inhibitors have synergistic effects with YAP in regulating cell cycle, increasing G1/S phase arrest. YAP, yesassociated protein; $M E K, M A P K /$ extracellular signal-regulated kinase. 

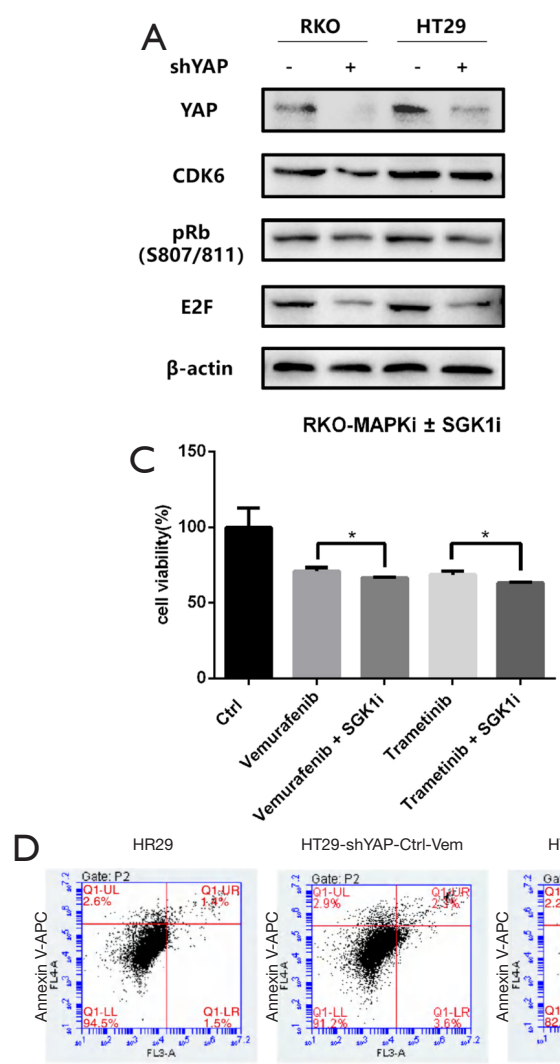

HT29-shYAP-Ctrl-Vem
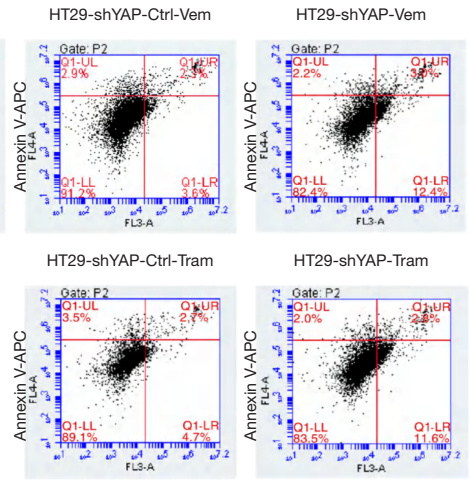

HT29-shYAP-Tram

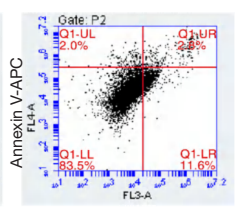

$\mathrm{E}$
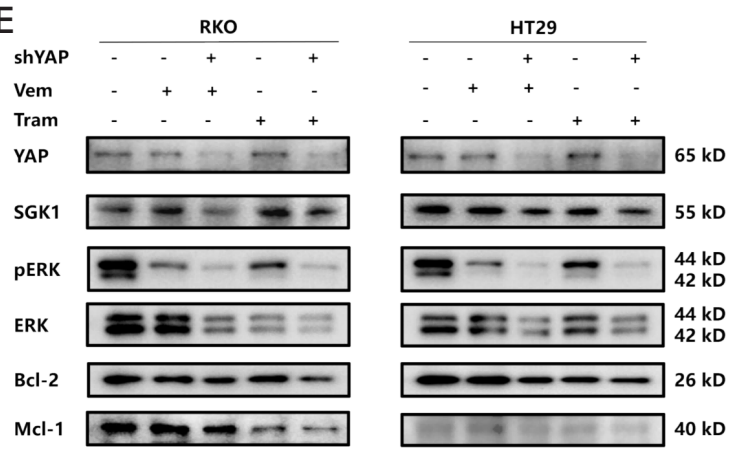
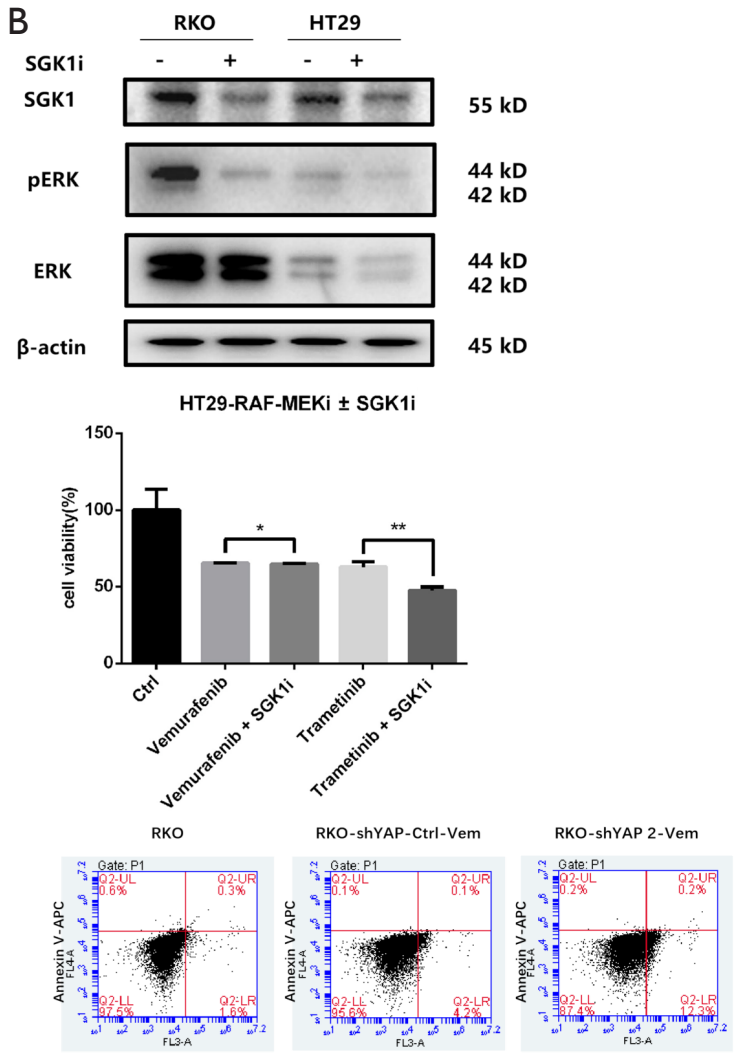

RKO-shYAP-Ctrl-Tram

RKO-shYAP 2-Tram
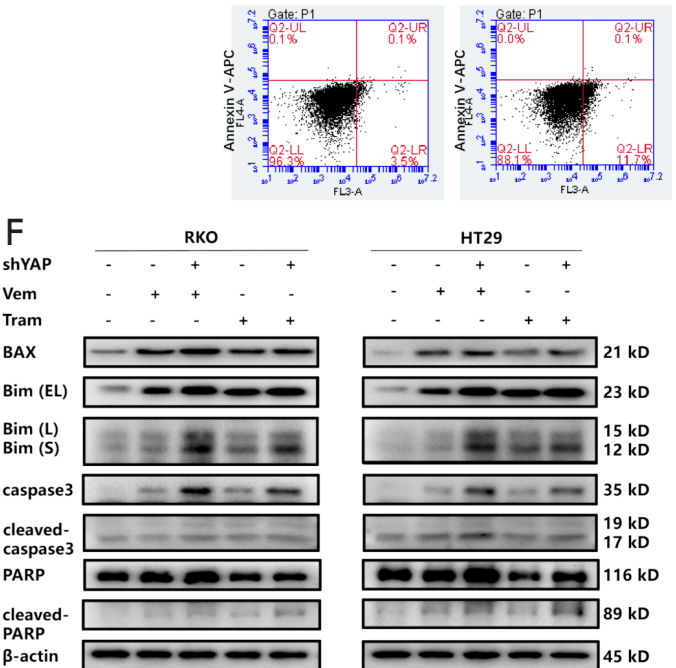

Figure 4 YAP regulates the response mechanism of $R A F$ - and $M E K$-targeted therapy. (A) $S G K 1$ protein levels were downregulated after YAP knockdown. (B) $S G K 1$ inhibition resulted in a significant decrease in $p E R K 1 / 2$ (activated form) expression, while the total ERK1/2 content showed no significant difference between the two groups. (C) Cell activity measurement: $S G K 1 i$ and $R A F \rightarrow M E K$ pathway inhibitor synergistic inhibition of the ERK1/2 pathway, inhibition of RKO, and HT29 cell proliferation. (D) Flow cytometry detection of apoptosis: Apoptosis induced by the $R A F \rightarrow M E K$ pathway I increased after YAP knockdown. (E,F) The $R A F M E K$ pathway-ERK pathway participates in cell apoptosis by regulating gene expression of $B c l-2$ family proteins, such as Bax Bim, and by inducing proteasomal degradation of anti-apoptotic proteins. WB detection knockdown of YAP reduces the expression level of SGK1, thereby reducing the expression of $p E R K$, coordinating the expression of apoptosis-related factors, and increasing the apoptosis induced by the $R A F \rightarrow M E K$ pathway i. YAP, yes-associated protein; $M E K$, MAPK/extracellular signal-regulated kinase. ${ }^{*} \mathrm{P}<0.01,{ }^{* *} \mathrm{P}<0.05$. 
for survival and again, that PFS was much shorter than in wild-type patients (3).

A mutated BRAF inhibitor drug curative effect is not possible because the feedback of inhibitors for $B R A F$ mutations leads to abnormal activation of the EGFR pathway and results in the continued proliferation of tumor cells. However, researchers have identified the feedback mechanism by targeting the $B R A F, M E K$, and EGFR pathways as blocking targets, including the $B R A F \mathrm{~V} 600 \mathrm{E}$ mutation, providing a novel treatment idea and opportunity.

The BRAF V600E small molecule inhibitor vemurafenib has a satisfactory effect on melanoma. A phase III clinical trial comparing vemurafenib and imidazole carboxamide (dacarbazine) showed that the optimal overall response rate in the BRAF $\mathrm{V} 600 \mathrm{E}$ mutated population was $48 \%$ in the Braf-inhibitor group, which was $>5 \%$ higher than in the imidazole carboxamide group (30). However, $B R A F$ inhibitors are not effective in patients with $B R A F$-mutant CRC. A study of 21 patients found that only one treated with a $B R A F$ inhibitor responded (5\%), while four others showed some improvement. Research on the mechanism of drug resistance in CRC has found that $B R A F$-inhibitor treatment, such as acne medication applied to CRC cell lines with $B R A F$ mutations, led to feedback that activated EGFR (31). Moreover, after BRAF is inhibited, CRAF and MARK downstream pathways can mediate EGFR toward fast reactivation, ultimately leading to drug resistance. Inhibiting multiple targets simultaneously may prevent this problem, providing a theoretical basis for a new treatment strategy (29).

$R A F$ and $M E K$ inhibitors are effective for most cancers, but targeted therapy is inhibited due to drug resistance. Studies have shown that YAP can promote both primary and acquired resistance to $R A F$ - and $M E K$-targeted therapies in cancer patients. In addition, the Hippo-YAP pathway can regulate cell proliferation and apoptosis as well as promote the development of tumor which means YAP is an effective target for tumor-targeted therapy $(32,33)$.

Recent research confirmed the upregulation of YAP was associated with cetuximab resistance in patients with CRC (3). In cancer patients with wild-type KRAS, patients without YAP activation may benefit from cetuximab treatment. These results suggest that YAP activation can be used as a target for EGFR inhibitor therapy and a prognostic marker for CRC patients (34). YAP expression has also been used as a marker of primary EGFR inhibitor resistance in pancreatic cancer and has been shown to be related to the high expression of prostaglandin E2, thereby affecting the occurrence and development of human colitisrelated cancers and CRC (35-38).

In this study, we verified for the first time that the transcription factor YAP was upregulated in BRAF V600E mutant CRC cells. Knocking down YAP reduced the growth rate, population dependence, proliferation, and invasion ability of colon cancer cells. Furthermore, YAP knockdown increased the sensitivity of BRAF V600E mutant CRC cells to MAPK pathway inhibitors. The mechanism underlying the regulation of $R A F$ - and $M E K$-targeted therapy response was YAP cooperation with $R A F \rightarrow M E K$ pathway inhibitors to regulate the cell cycle, as indicated by YAP knockdown increasing G1/S phase arrest. Previous studies have shown that two conserved TEAD binding sites exist in the CDK6 promoter. The detection of YAP-TEAD binding to these two sites by ChIP and qPCR confirmed that CDK6 is the downstream target gene of the YAP-TEAD complex, and that YAP affects $C D K 6$ expression. In addition, transcriptomics and differential gene expression analyses were performed on HT29 cells expressing wild-type YAP and shYAP revealing the $S G K 1$ gene was significantly downregulated under the action of shYAP. ChIP and qPCR confirmed that $S G K 1$ is transcriptionally activated by the YAP-TEAD complex and the downstream target gene and YAP were seen to knock down the $S G K 1$ protein level down. Existing studies have shown that $S G K 1$ is an activator of $E R K 1 / 2$, which in turn regulates the $E R K 1 / 2$ pathway. We verified that $S G K 1$ inhibition resulted in a significant decrease in the expression of $p E R K 1 / 2$ (activated form), while the total amount of ERK1/2 was not significantly different between the two groups. Finally, YAP can further affect $p E R K$ expression through influencing $S G K 1$, coordinating the expression of apoptosis-related factors, and increasing apoptosis induced by $R A F \rightarrow M E K$ pathway inhibition. Thus, our work has contributed to research on the role of YAP in promoting resistance of $B R A F \mathrm{~V} 600 \mathrm{E}$ mutant CRC cells to MAPK pathway inhibitors. An increase in YAP protein expression among BRAF V600E patients with metastatic CRC is a biomarker of poor initial response and acquired resistance to $R A F-M E K$ inhibitors. Taken together, these patterns suggest that YAP inhibition combined with $R A F-M E K$ inhibitors is a potentially effective method to overcome drug resistance, and improve patient response and survival.

\section{Conclusions}

Our results show that YAP can promote the primary drug 
resistance of MAPK pathway inhibitors in metastatic CRC with the $B R A F$ V600E mutation. Identification of the mechanism of drug resistance can provide a novel method and treatment strategy for metastatic CRC harboring the $B R A F$ V600E mutation to overcome drug resistance and improve patient survival.

\section{Acknowledgments}

Funding: This study was supported by grants from the Science and Technology Planning Project of Shenyang (No. 191124088); Science and Technology Planning Project of Liaoning Province of China (No. 20180530092); National Key R\&D Program of China (Grant \#2018YFC1311600); Scientific research foundation for the introduction of talents, Liaoning Cancer Hospital \& Institute (No. Z1702); and Science and Technology Planning Project of Shenyang (No. 191124086).

\section{Footnote}

Reporting Checklist: The authors have completed the MDAR reporting checklist. Available at https://dx.doi. org/10.21037/jgo-21-258

Data Sharing Statement: Available at https://dx.doi. org/10.21037/jgo-21-258

Conflicts of Interest: All authors have completed the ICMJE uniform disclosure form (available at https://dx.doi. org/10.21037/jgo-21-258). The authors have no conflicts of interest to declare.

Ethical Statement: The authors are accountable for all aspects of the work in ensuring that questions related to the accuracy or integrity of any part of the work are appropriately investigated and resolved.

Open Access Statement: This is an Open Access article distributed in accordance with the Creative Commons Attribution-NonCommercial-NoDerivs 4.0 International License (CC BY-NC-ND 4.0), which permits the noncommercial replication and distribution of the article with the strict proviso that no changes or edits are made and the original work is properly cited (including links to both the formal publication through the relevant DOI and the license). See: https://creativecommons.org/licenses/by-nc$\mathrm{nd} / 4.0 /$.

\section{References}

1. Siegel RL, Miller KD, Jemal A. Cancer Statistics, 2017. CA Cancer J Clin 2017;67:7-30.

2. Siegel RL, Miller KD, Fedewa SA, et al. Colorectal cancer statistics, 2017. CA Cancer J Clin 2017;67:177-93.

3. Kopetz S, Grothey A, Yaeger R, et al. Encorafenib, Binimetinib, and Cetuximab in BRAF V600E-Mutated Colorectal Cancer. N Engl J Med 2019;381:1632-43.

4. Lin L, Sabnis AJ, Chan E, et al. The Hippo effector YAP promotes resistance to RAF- and MEK-targeted cancer therapies. Nat Genet 2015;47:250-6.

5. Wang K, Li Y, Song N, et al. Signal transducer and activator of transcription 3 inhibition enhances vemurafenib sensitivity in colon cancers harboring the BRAFV600E mutation. J Cell Biochem 2019;120:5315-25.

6. Janku F. Advances on the BRAF Front in Colorectal Cancer. Cancer Discov 2018;8:389-91.

7. Chan XY, Singh A, Osman N, et al. Role Played by Signalling Pathways in Overcoming BRAF Inhibitor Resistance in Melanoma. Int J Mol Sci 2017;18:1527.

8. Choe MH, Yoon Y, Kim J, et al. miR-550a-3-5p acts as a tumor suppressor and reverses $\mathrm{BRAF}$ inhibitor resistance through the direct targeting of YAP. Cell Death Dis 2018;9:640.

9. Zanconato F, Piccolo S. Eradicating tumor drug resistance at its YAP-biomechanical roots. EMBO J 2016;35:459-61.

10. Kim HB, Myung SJ. Clinical implications of the HippoYAP pathway in multiple cancer contexts. BMB Rep 2018;51:119-25.

11. Flaherty KT, Wargo JA, Bivona TG. YAP in MAPK pathway targeted therapy resistance. Cell Cycle 2015;14:1765-6.

12. Liu BS, Xia HW, Zhou S, et al. Inhibition of YAP reverses primary resistance to EGFR inhibitors in colorectal cancer cells. Oncol Rep 2018;40:2171-82.

13. Lulla AR, Slifker MJ, Zhou Y, et al. miR-6883 Family miRNAs Target CDK4/6 to Induce G1 Phase Cell-Cycle Arrest in Colon Cancer Cells. Cancer Res 2017;77:6902-13.

14. Pek M, Yatim SMJM, Chen Y, et al. Oncogenic KRASassociated gene signature defines co-targeting of CDK4/6 and MEK as a viable therapeutic strategy in colorectal cancer. Oncogene 2017;36:4975-86.

15. Chen SH, Gong X, Zhang Y, et al. RAF inhibitor LY3009120 sensitizes RAS or BRAF mutant cancer to CDK4/6 inhibition by abemaciclib via superior inhibition of phospho-RB and suppression of cyclin D1. Oncogene 2018;37:821-32. 
16. Kim M, Kim T, Johnson RL, et al. Transcriptional corepressor function of the hippo pathway transducers YAP and TAZ. Cell Rep 2015;11:270-82.

17. Li Z, Razavi P, Li Q, et al. Loss of the FAT1 Tumor Suppressor Promotes Resistance to CDK4/6 Inhibitors via the Hippo Pathway. Cancer Cell 2018;34:893-905.e8.

18. Miller DM, Flaherty KT. Cyclin-dependent kinases as therapeutic targets in melanoma. Pigment Cell Melanoma Res 2014;27:351-65.

19. Conza D, Mirra P, Calì G, et al. The SGK1 inhibitor SI113 induces autophagy, apoptosis, and endoplasmic reticulum stress in endometrial cancer cells. J Cell Physiol 2017;232:3735-43.

20. Liu H, Yu J, Xia T, et al. Hepatic serum- and glucocorticoid-regulated protein kinase 1 (SGK1) regulates insulin sensitivity in mice via extracellular-signal-regulated kinase 1/2 (ERK1/2). Biochem J 2014;464:281-9.

21. Sullivan RJ. Dual MAPK/CDK Targeting in Melanoma: New Approaches, New Challenges. Cancer Discov 2018;8:532-3.

22. Towhid ST, Liu GL, Ackermann TF, et al. Inhibition of colonic tumor growth by the selective SGK inhibitor EMD638683. Cell Physiol Biochem 2013;32:838-48.

23. Wang YF, Jiang CC, Kiejda KA, et al. Apoptosis induction in human melanoma cells by inhibition of MEK is caspaseindependent and mediated by the Bcl-2 family members PUMA, Bim, and Mcl-1. Clin Cancer Res 2007;13:4934-42.

24. Won M, Park KA, Byun HS, et al. Protein kinase SGK1 enhances MEK/ERK complex formation through the phosphorylation of ERK2: implication for the positive regulatory role of SGK1 on the ERK function during liver regeneration. J Hepatol 2009;51:67-76.

25. Yoo G, Kim T, Chung C, et al. The novel YAP target gene, SGK1, upregulates TAZ activity by blocking GSK3 mediated TAZ destabilization. Biochem Biophys Res Commun 2017;490:650-6.

26. Bai JA, Xu GF, Yan LJ, et al. SGK1 inhibits cellular apoptosis and promotes proliferation via the MEK/ ERK/p53 pathway in colitis. World J Gastroenterol 2015;21:6180-93.

27. Zhang P, Kawakami H, Liu W, et al. Targeting CDK1 and MEK/ERK Overcomes Apoptotic Resistance in BRAFMutant Human Colorectal Cancer. Mol Cancer Res 2018;16:378-89.

28. Zhu L, Dai LM, Shen H, et al. Qing Chang Hua Shi granule ameliorate inflammation in experimental rats and cell model of ulcerative colitis through MEK/
ERK signaling pathway. Biomed Pharmacother 2019;116:108967.

29. Corcoran RB, André T, Atreya CE, et al. Combined BRAF, EGFR, and MEK Inhibition in Patients with BRAFV600E-Mutant Colorectal Cancer. Cancer Discov 2018;8:428-43.

30. Martin CA, Cullinane C, Kirby L, et al. Palbociclib synergizes with BRAF and MEK inhibitors in treatment naïve melanoma but not after the development of BRAF inhibitor resistance. Int J Cancer 2018;142:2139-52.

31. Prahallad A, Sun C, Huang S, et al. Unresponsiveness of colon cancer to BRAF(V600E) inhibition through feedback activation of EGFR. Nature 2012;483:100-3.

32. Lin L, Bivona TG. The Hippo effector YAP regulates the response of cancer cells to MAPK pathway inhibitors. Mol Cell Oncol 2016;3:e1021441.

33. Dasgupta I, McCollum D. Control of cellular responses to mechanical cues through YAP/TAZ regulation. J Biol Chem 2019;294:17693-706.

34. Nakamura Y, Hattori N, Iida N, et al. Targeting of superenhancers and mutant BRAF can suppress growth of BRAF-mutant colon cancer cells via repression of MAPK signaling pathway. Cancer Lett 2017;402:100-9.

35. Ma K, Xu Q, Wang S, et al. Nuclear accumulation of Yes-Associated Protein (YAP) maintains the survival of doxorubicin-induced senescent cells by promoting survivin expression. Cancer Lett 2016;375:84-91.

36. Theodosakis N, Langdon CG, Micevic G, et al. Inhibition of isoprenylation synergizes with MAPK blockade to prevent growth in treatment-resistant melanoma, colorectal, and lung cancer. Pigment Cell Melanoma Res 2019;32:292-302.

37. Xie Q, Chen J, Feng H, et al. YAP/TEAD-mediated transcription controls cellular senescence. Cancer Res 2013;73:3615-24.

38. Zhao X, Wang X, Fang L, et al. A combinatorial strategy using YAP and pan-RAF inhibitors for treating KRASmutant pancreatic cancer. Cancer Lett 2017;402:61-70.

(English Language Editor: B. Draper)

Cite this article as: Su M, Zhan L, Zhang Y, Zhang J. Yesactivated protein promotes primary resistance of $B R A F \mathrm{~V} 600 \mathrm{E}$ mutant metastatic colorectal cancer cells to mitogen-activated protein kinase pathway inhibitors. J Gastrointest Oncol 2021;12(3):953-963. doi: 10.21037/jgo-21-258 